\title{
Robot Movement Uncertainty Determines Human Discomfort in Co-worker Scenarios
}

\author{
Daphné Héraïz-Bekkis CNRS-AIST \\ JRL UMI3218/RL Tsukuba, Japan \\ University of Montpellier Montpellier, \\ France \\ e-mail:daphne.hbek.info@gmail.com \\ Eiichi Yoshida \\ CNRS-AIST JRL UMI3218/RL \\ Tsukuba, Japan \\ e-mail: e.yoshida@aist.go.jp \\ CNRS-UM LIRMM UMR5506 \\ Montpellier, France \\ CNRS-AIST JRL UMI3218/RL \\ Tsukuba, Japan \\ e-mail: \\ ganesh.gowrishankar@lirmm.fr
}

\author{
Natsuki Yamanobe \\ ISRI/AIRC \\ AIST \\ Tsukuba/Tokyo, Japan \\ e-mail: \\ n-yamanobe@aist.go.jp
}

\begin{abstract}
The long term success of a human-robot interaction will depend on how comfortable and safe a human feels with it. But which feature of a robot's movement determines human comfort? To address this question, here we considered four different models of human discomfort. We then designed an empirical human-robot co-worker task that enables us to both, quantify the discomfort experienced by the human co-worker by analyzing behavioral changes, and examine which model of discomfort explains the changes best. Using this task, we show that the perceived uncertainty in a robot's movement is a key determinant of human discomfort, and we discuss how movement uncertainty can give a unified explanation for the modulation of human comfort with robots, and trust in them, as reported in several previous studies.
\end{abstract}

Keywords-human robot interaction; comfort; robot predictability; movement uncertainty; human perception

\section{INTRODUCTION}

Modern robots are required to work increasingly close to humans in co-worker scenarios where the robot has to often share the workspace with a human. To ensure the acceptance of robots in these scenarios, it is important to ensure safe humanrobot interactions. Several studies have addressed this issue from an engineering perspective by trying to see how a robot should be designed [1] [2], controlled [3] [4] [5] or moved [6] [7] to ensure the safety of the human co-worker. However, fewer studies have looked at the human perspective during these interactions, to see what behaviors are perceived safe and comfortable by the humans. Human-robot interaction studies have often tried to measure the 'trust' felt by the humans towards robots [8] [9] [10] [11]. While the terminology of trust has been used broadly to explain various aspects of human emotions during robot interactions [10], it is arguably a highly cognitive emotion, which is developed by an individual towards an interacting partner, after explicit analysis of the partner's physical as well as task performance characteristics. In case of a robot, this can include how human it looks, its performance reliability, performance ability and faults [12] [13], sometime varying heavily with cultural factors [9]. On the other hand, here we are interested in the more implicit and automatic feeling that is induced by the presence of a

This work was supported by JSPS KAKENHI Grant Number JP17H01804. GG was partially supported by JST ERATO Grant Number JPMJER1701. robot near a human. By 'comfort' (or rather 'discomfort'), we refer to arousal with negative valence, related to anxiety and fear, in a human when in the vicinity of a robot. An example of this discomfort is the feeling that we have all experienced in a crowded train when an unknown individual stands too close to us. This discomfort makes us want to move away from the person. The discomfort is stronger when the person is not just standing nearby, but is dancing to the music from his headphones, and would be arguably even stronger if the moving human individual is replaced by a moving robot. Here, first, we are interested in understanding what aspect of the person's (or robot's) movement causes this discomfort. Second, while previous studies have explored similar basic emotions in the presence of robots [14] [15] utilizing cognitive scoring methods to quantify the human emotions, we aim to quantify the comfort by a behavioral measure- this we believe is crucial for an automatic emotion, that we believe discomfort is.

The discomfort mentioned in the example of the crowded train is quite probably caused by the fear that the person or robot may collide with us. It is thus obvious that discomfort is perceived only when a robot is close enough to collide with us, and correspondingly discomfort is minimal if the robot is very far away. Thus here, we are interested in robot-human interaction scenarios where a collision is possible. In such a scenario, our main goal is to isolate the key aspect of robot behavior that determines human discomfort. To answer this question, we devised four qualitative models of human discomfort. Each model is motivated by previous human behavioral studies and our intuition, and considers one behavioral attribute (of the robot) that may be a key determinant of human comfort. We then designed an empirical industrial human-robot interaction task and examined which of our model can explain the human discomfort in the task best.

\section{A. Discomfort Models}

Before defining our comfort models, we start by defining the undisturbed human trajectory (UHT) as the human movement trajectory when performing his/her task without disturbance from the robot co-worker -for example, when the robot is sufficiently far away. Then, our first robot-proximity $(R P)$ 
model of discomfort assumes that the proximity of a robot to a human's undisturbed movement trajectory is the key determinant of how comfortable the human is with it. This model is inspired by the numerous human studies that have shown that during movements, humans can isolate the key features in their environment (usually edges of obstacles) that are most likely to cause a collision during human movements [16], and focus their visual attention on these points. In our study, we hypothesized this key feature to be the location of the tip of the robot end-effector (see our chosen task in the methods section),every time it approached the human.

Let $p_{t}$ represent the closest distance of the robot end effector to the UHT at any time $t$ and let $P_{j}$ represent the set of closest distances of the robot end effector to the UHT over the $j^{t h}$ human trial. Then the discomfort of the human co-worker in the trial $k$ with the robot, represented by the variable $\delta_{k}$, is given in the RP model by:

$$
\delta_{k}^{R P}=\gamma^{R P}\left(1-\frac{1}{\rho} \Omega_{j=1}^{k-1}\left[\min \left(P_{j}\right)\right]\right)
$$

where $\Omega_{k 1}^{k 2}[x]$ represents the statistical average of recordings of the variable $\mathrm{x}$ in across the trials $j=[k 1, k 2] . \min ()$ represents the minimum value function and $\rho$ corresponds to the maximum value of the numerator and ensures that the right hand side is constrained within $[0,1] . \gamma$ is an unknown proportionality constant between the variable on the right hand side of the equation to the discomfort $\delta_{k}$.

Second, the robot velocity $(R V)$ model considers the robot velocity to be the key determinant of human discomfort, and that a human feels more discomfort when the average velocity of a co-worker robot is faster. This model is motivated by intuition and by visual studies that show that our attention is attracted by large velocities of observed objects. The RV model can thus be represented by:

$$
\delta_{k}^{R V}=\gamma^{R V} \Omega_{j=1}^{k-1}\left[\left|\dot{P}_{j}\right|\right] .
$$

Next, the robot range $(R R)$ model considers that the discomfort is determined fundamentally by the range of the robot movement. That is, if the movement range of the robot is large, the discomfort is more. This model is motivated by the intuition that, provided a robot is not very slow, larger movements are scarier to humans than smaller movements. The RR model thus assumes that

$$
\delta_{k}^{R R}=\gamma^{R R} \Omega_{j=1}^{k-1}\left[\max \left(P_{j}\right)-\min \left(P_{j}\right)\right] .
$$

Finally, the robot uncertainty (RU) model assumes that the discomfort towards a robot is determined by how close the robot comes to the UHT, but that the discomfort is modulated by the perceived uncertainty in this value, and not its average (like in the RP model). Implying, the discomfort of human coworkers is increased when the uncertainty of how close the robot will come to them increases. It is well know that interactive human behaviors are guided by movement predictions of their human [17] [18], as well as robot partners [19] [20] [14]. It can thus be considered that the behaviors are expected to be influenced when the partner movements are less predictable, and hence uncertain. Studies in social neuroscience show that the trust we feel towards an interacting agent is determined by our perceived uncertainty of their decision making behavior during monetary and social transactions [21]. Uncertainty, in regard to failures and errors by a robot has also shown to influence the trust towards it [11] [22] [23]. The RU model extends this idea to movement (rather than decision or performance) uncertainty and in regard to comfort rather than trust. The RU model assumes the standard deviation (STD) of a movement as a measure of the perceived uncertainty and can be represented as:

$$
\delta_{k}^{R U}=\gamma^{R U} S T D_{j=1}^{k-1}\left[\min \left(P_{j}\right)\right]
$$

Note that, as the values of $\gamma^{R P}, \gamma^{R V}, \gamma^{R R}$ and $\gamma^{R U}$ are not known, these models do not allow us to analyze the discomfort quantitatively. However, as we will show later, the models still allow us to qualitatively determine the key behavioral determinant of discomfort.

\section{MethodS}

We developed an empirical human-robot co-worker task and utilized different robot behaviors in this task to evaluate which of the four model can best explain the discomfort observed in the human partners. Our task required human participants to move their hand from a start, to a goal to a target line on a table (explained in detail later). They move in the vicinity of a robot that moves back and forth making its own reach movements near the UHT of the participants. We chose an arm reaching task as it is typical of experiments in motor neuroscience [24] [25] [26] where multiple studies have shown that behaviors and models isolated in reaching studies are representative of whole body behaviors [24] [25], with applications in robot human interaction, including rehabilitation [26] [27] [28] [29].

\section{A. Participants}

21 participants (15 males, 6 females of 14 nationalities, aged 20-43, mean 27.9 \pm SD 6.32) participated in our experiment. One of them was left-handed. They were recruited through online advertisements, via SNS or mailing lists related to the city where our institute is. They had several backgrounds such as university students, homemakers, researchers, engineers, etc. The experiments were approved by the local ethics committee at the National Institute of Advanced Industrial Science and Technology (AIST), Japan. All the participants gave informed consent to participate in the study. All participants were naive to the motive of the experiment. They were compensated up $¥ 1500 \mathrm{JPY} /$ hour for their time.

\section{B. Experiment Setup}

Our experiment setup is shown in Fig. 1 and Fig. 2(a). The participants sat comfortably on a chair in front of a large table $(120 \times 60 \times 70 \mathrm{~cm})$. A starting point and a goal line were presented on the table. The start point, of $1.5 \mathrm{~cm}$ diameter, was drawn $170 \mathrm{~mm}$ from the edge of the table, close to the participant. The goal line, $630 \mathrm{~mm}$ long, $40 \mathrm{~mm}$ wide, had its lower edge $150 \mathrm{~mm}$ away from the start point. Each participant 


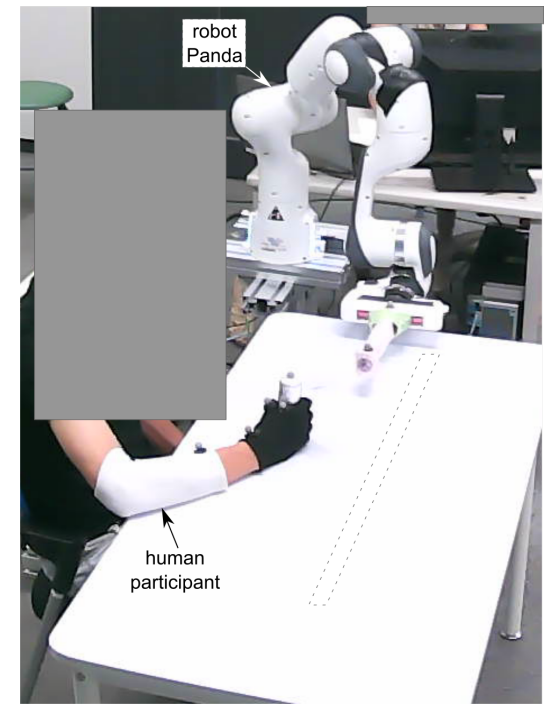

Figure 1. Photo of the setup with a participant: Due to the light reflection, the target line is not visible and thus has been highlighted with a black dotted line on the photo. Here, the participant is shown at the starting point.

was asked to wear an elbow support and a finger-less glove while holding a $40 \mathrm{~mm}$ diameter, $120 \mathrm{~mm}$ high stylus.

We used a 7 degrees of freedom robot (Franka Emika Panda) for our experiment. The robot was installed on a stand $(715$ $\mathrm{mm}$ high) in the left-side corner of the table with respect to the human participant. We attached a $230 \mathrm{~mm}$ long and 35 $\mathrm{mm}$ diameter padded cardboard stick to the robot gripper to ensure that any collision with the participant is safe and soft. We will refer to this stick as the robot end effector (EE).

We adopted a camera-based motion capture system to measure the human and robot motions. In total, seven passive reflective $\mathrm{m}$ arkers $\mathrm{w}$ ere $\mathrm{p}$ laced o $\mathrm{n} t$ he $\mathrm{r}$ ight $\mathrm{f}$ orearm, wrist, hand of a participant, on the tip of the robot stick, the human stylus, and on the gripper of the robot. These were tracked using seven kestrel infra-red cameras (Motion Analysis Co.) at $200 \mathrm{~Hz}$ (for 5 participants the recordings were done at $100 \mathrm{~Hz}$ due to a system error). We will focus our analysis on the markers on the human stylus, and the tip of the robot EE.

\section{Task}

Experiments were conducted by two experimenters, one managing the experiment process and the other managing the systems. Before the experiments, participants were provided with a overview of the task and procedures with their consent form. Then, they were asked to enter the experiment room where the setup was. After they sat at the table, the experimenter explained the task to the participants. The experiment was conducted with one participant at a time.

As mentioned before, our experiment required the participants to make repeated reaching movements from a given start point, holding a stylus, to a target (see Fig. 2(a)). We performed two experiments, one in which the target was a point, and one in which it as a line. Due to constraints in the maximum speed of our robot (relative to the speed of

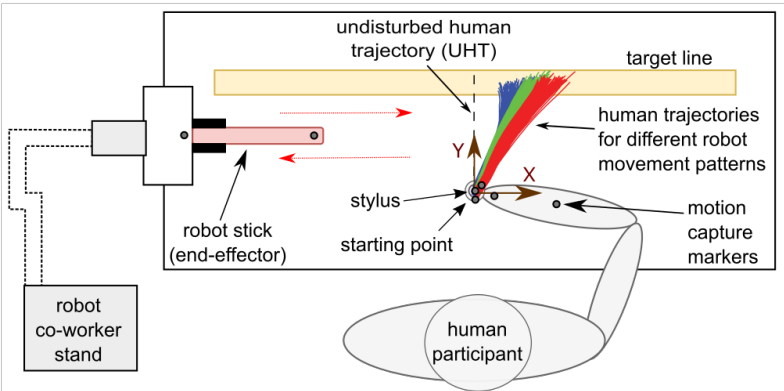

(a) Top view of the setup of the experiment

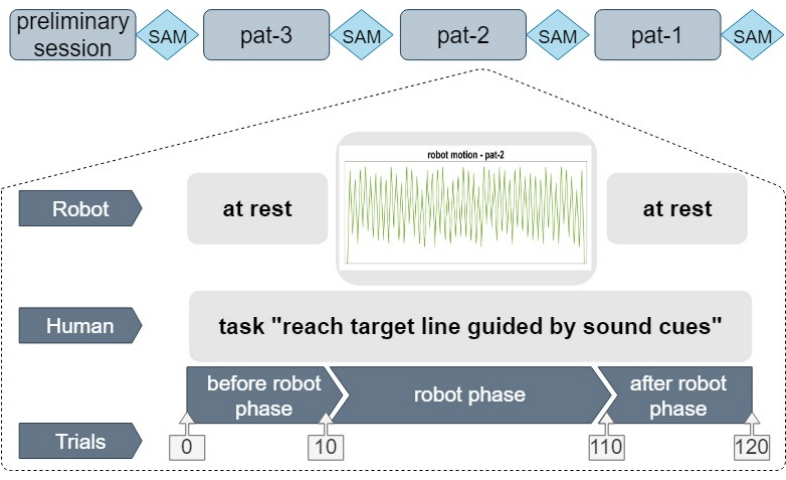

(b) Detailed timeline of the experiment

Figure 2. Experimental design: (a) Diagram of the experiment setup shows the relative position of the human and robot, the position of the motion tracking markers and the coordinate frame used in the data analysis. The movement performed by one participant, for the three different robot movement patterns (in different colours), have been shown superimposed on the figure. (b) Timeline of the experiment with an arbitrary order of robot movement patterns. The participants worked in four sessions. Each session was divided into three phases (of 10, 100 and 10 trials), and the robot movement in the robot phase was different between the four sessions. 'SAM' represents the questionnaire scored by the participants in between the sessions.

human reach), we found that the point reach experiment could not induce sufficient discomfort in participants. We therefore concentrate on the line reach experiment here. The participants were asked to sit in front of the table such that the start point was in front of the center of their chest. They were instructed to always hold the stylus so that both their hand and the stylus were touching the table, and to "always slide the stylus on the table and never lift the arm during the experiment". They were asked to make a 'one shot' reaching movement (without stops) to the target line while avoiding bumping into the robot. No instructions were given about where to reach on the line and the participants chose and change their reach point (on the target line) freely through the experiments.

A repetitive pattern of three high pitched beeps (first two beeps separated by $70 \mathrm{~ms}$ and the last separated by $200 \mathrm{~ms}$ ) were utilized to instruct the participants on when to start and complete their reaches. The participants were instructed to "get ready on the first beep, start moving on the second, and move fast enough to reach the target line by the end of the third beep; then return back to the start point before the next first beep". The first beep (hence the beep pattern) repeated every one second. We designed the beep timings considering 
two issues. First, to ensure that the participants make oneshot and straight reaches (we used preliminary experiments to converge to this beep time). Second, to synchronize the human movement with the start of the robot movement, such that the robot would sufficiently interfere and potentially cause them discomfort. Participants were trained as long as they needed, till they felt they could match the sound cues with ease. They were specifically told to find a comfortable body posture for the task and then keep it through the experiment. Before the start of each session, the participants were again reminded to focus on the sound cues while performing the task.

Fig.2(b) shows the timeline of our experiment. The participants worked in four sessions, each 2.5 minutes long. Each session consisted of three phases. In each session, the participants started with 10 round-trip trials while the robot remained stationary at a distance of $170 \mathrm{~mm}$ from the UHT (before phase), followed by 100 round-trip trials with the robot moving (robot-phase) along the $\mathrm{X}$ axis, at distance of $90 \mathrm{~mm}$ along the $\mathrm{Y}$ axis from their start point (see Fig. 2(a) and later explanation of robot movements), and finally by 10 round-trip trials when the robot was again motionless (after robot phase). A low pitch sound cue was used to inform the participants of the change of phase. The robot movement in the robot-phase was different among the four sessions and these differences are explained below.

After each session, the participants were asked to answer the Self-Assessment Manikin (SAM) [30] survey on the session. The SAM picture-based test assess the participants emotional state (specifically 'happiness', 'anxiety' and 'feeling of control') during the experiment. We later converted the chosen pictures to a numerical scale going from -4 to 4 , with +4 indicating high values of the state.

The participants were also given an open-ended question, where they were allowed to report any concerns, thoughts and feelings about the experiment session.

\section{Robot Movement Patterns}

As mentioned before, the robot movement was different in the robot-phase of the four sessions.

The robot movements were restricted to the plane of the table, and along the $X$ axis (see coordinates in Fig. 2(a)) in all the sessions. In each session, the robot performed to and fro movement cycles defined by three parameters which are the minimum distance of the robot EE from the UHT $\left(d_{\min }^{U H T}\right)$ in each movement, the maximum distance of the robot EE from the UHT $\left(d_{\max }^{U H T}\right)$ in each movement, and the variation $\left(d_{\text {variation }}\right)$ in the values of $d_{\min }^{U H T}$ and $d_{\max }^{U H T}$ across the session.

The first session served as a preliminary session to accustom the participants in following the cues while working in the proximity of the disturbing robot. The preliminary session thus used the parameters of $d_{\min }^{U H T}=60 \mathrm{~mm}, d_{\max }^{U H T}=140 \mathrm{~mm}$ and $d_{\text {variation }}=0 \mathrm{~mm}$. That is, the robot moved between $140 \mathrm{~mm}$ and $60 \mathrm{~mm}$ of the UHT in every movement cycle.

Following the preliminary session, the participants worked in three experimental sessions that we will analyze in detail

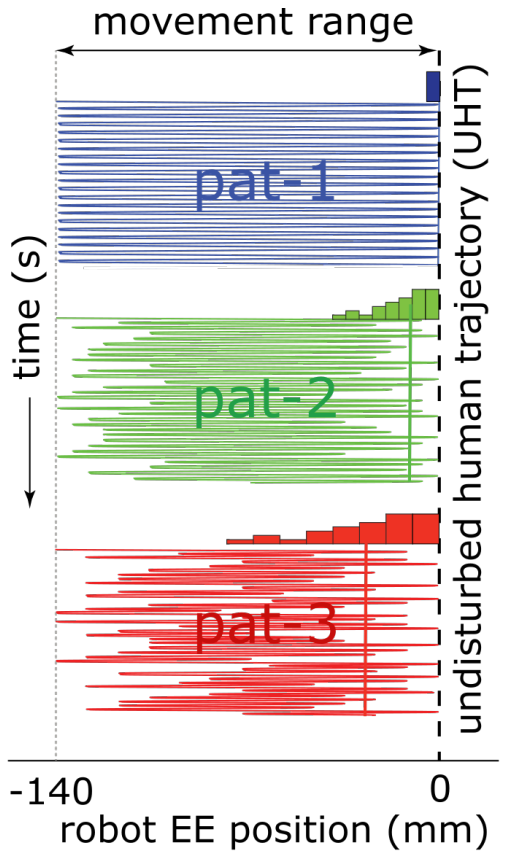

Figure 3.Robot patterns: Robot EE movements, in the $\mathrm{X}$ axis, for each of the three patterns, with respect to the UHT. Histograms show the distribution of the minimum distance of approach $\left(d_{\mathrm{min}}^{U H T}\right)$ in each pattern. The vertical coloured lines on the patterns represent the mean value of $d_{\min }^{U H T}$.

next. The robot followed three movement patterns (pat-1, pat-2 and pat-3), one each in a session. These movement patterns were specifically designed to enable us to qualitative distinguish the key determinant of discomfort utilizing our four discomfort models. The order of sessions with pat-1, pat 2 and pat-3 was balanced across the participants.

Pat-1 is shown as a blue trace in Fig. 3. It utilized the parameters of $d_{\min }^{U H T}=0 \mathrm{~mm}, d_{\max }^{U H T}=140 \mathrm{~mm}$ and $d_{\text {variation }}$ $=0 \mathrm{~mm}$. This indicates that the robot movements were repetitive in pat-1, and the robot moved between $140 \mathrm{~mm}$ and $0 \mathrm{~mm}$ of the UHT in every movement cycle of this pattern.

Pat-2 is shown in green in Fig. 3. Pat-2 utilized the parameters of $d_{\min }^{U H T}=0 \mathrm{~mm}, d_{\max }^{U H T}=140 \mathrm{~mm}$ and $d_{\text {variation }}=40$ $\mathrm{mm}$. In this movement pattern, while the robot stayed within $140 \mathrm{~mm}$ and $0 \mathrm{~mm}$ of the UHT, there were variations up until $40 \mathrm{~mm}$ of $d_{\min }^{U H T}$ and $d_{\max }^{U H T}$ across movement cycles. $d_{\min }^{U H T}$ varied between 0 and $40 \mathrm{~mm}$, with the individual variations chosen pseudo-randomly from a half-normal distribution (with the fold at $d_{\min }^{U H T}=0$ and standard deviation $=11.5 \mathrm{~mm}$, see distribution of $d_{\min }^{U H T}$ shown in green above pat-2 in Fig. 3). Similarly, $d_{\max }^{U H T}$ varied between $140 \mathrm{~mm}$ and $100 \mathrm{~mm}$, with variations again chosen from a half-normal distribution (this time with the fold at $d_{\max }^{U H T}=140$ and standard deviation $=$ $11.5 \mathrm{~mm})$.

Finally, Pat-3 (see red trace in Fig. 3) utilized the parameters of $d_{\min }^{U H T}=0 \mathrm{~mm}, d_{\max }^{U H T}=140 \mathrm{~mm}$ and $d_{\text {variation }}=80 \mathrm{~mm}$. As well as for pat-2, while the robot stayed within $140 \mathrm{~mm}$ and $0 \mathrm{~mm}$ of the UHT, in pat-3, there were variations up until $80 \mathrm{~mm}$ across movement cycles in the values of $d_{\min }^{U H T}$ 


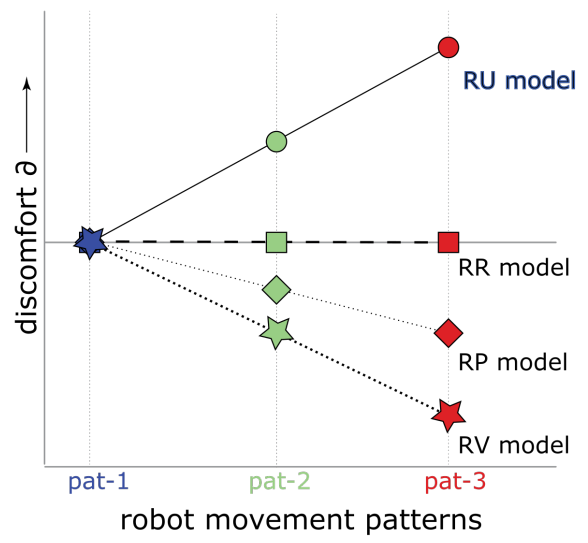

Figure 4. Discomfort model predictions: The predicted discomfort $\delta$ by our four models with the three robot patterns.

and $d_{\max }^{U H T}$. These variations were chosen from a half-normal distribution, this time with the standard deviation $=23 \mathrm{~mm}$ see the distribution of $d_{\min }^{U H T}$ shown in red above pat-3 in Fig. $3)$.

\section{E. Model Predictions}

Given the three patterns of robot movement, we next utilized the equations 1 to 4 and calculated the discomfort estimated by each of our four model for the three patterns of robot movement. These estimates are shown in Fig. 4. We assume an appropriate value for $\gamma^{R P}, \gamma^{R V}, \gamma^{R R}$ and $\gamma^{R U}$ so as to scale the model predictions in Fig. 4. Note again that these $\gamma$ values are not important, as we are interested in the qualitative predictions by these models.

The lowest value of $d_{\mathrm{min}}^{U H T}$ was zero in all the three patterns showing that the robot approaches up till the UHT in all the patterns. However, in pat-1, the robot reaches the UHT in every movement cycle, whereas in pat-2 and pat-3, it approaches the UHT in fewer cycles (compare blue, green and red distributions in Fig. 3). The average minimal distance of the robot from the UHT is thus the smallest (equal to zero, see blue vertical line in Fig. 3) in pat-1. It is higher in pat-2 (at 13 $\mathrm{mm}$, see green vertical line in Fig. 3), maximum in pat-3 (at 26 $\mathrm{mm}$, see red vertical line in Fig. 3). Therefore, the RP model predicts that the discomfort is highest for pat-1, less for pat-2 and the least for pat-3 (see diamond trace in Fig. 4). Even though our robot moves with the same peak speed in all the patterns, it remains at the peak speed for longer time intervals in pat-1 (as it moves for longer distances). The mean speed is thus highest for pat-1. The RV model thus also predicts the highest discomfort for pat-1, less for pat-2 and the least for pat-3 (see star trace in Fig. 4). In all the robot movement patterns, the robot moves within the same movement range across a session (of $140 \mathrm{~mm}$, see Fig. 3). The RR model thus predicts that the discomfort will not change across the three robot patterns (see square trace in Fig. 4). Finally, considering again the minimal distance of the robot from the UHT, this value is same (and equal to zero) for every cycle in pat-

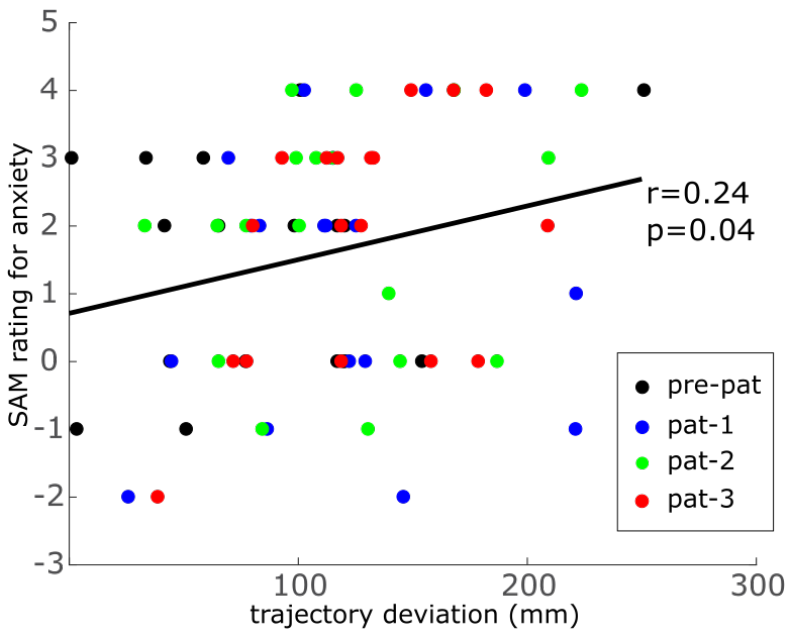

Figure 5. Questionnaire vs trajectory deviation: We observed a significant correlation between the median trajectory deviation by participants in the robot phase, and the anxiety level expressed by them in the SAM picture-based questionnaire (anxiety level increases from -4 to 4). The black trace represents the regression line.

1 , making it very predictable. The variation in the minimal distance is more in pat-2 (with a standard deviation, or std, = $11.5 \mathrm{~mm}$, green distribution in Fig. 3), and maximum in pat-3 $(\mathrm{std}=23 \mathrm{~mm}$, red distribution in Fig. 3) making the robot to be perceived as most uncertain in pat-3. The RU model, which looks at the STD of the minimal distance, thus predicts that the discomfort is minimal for pat-1, and progressively more for pat-2 and maximal for pat-3 (see circle trace in Fig. 4).

Overall, the four models provide different qualitative predictions (though the predictions are bit similar for the RP and RV models) of the human discomfort across our three sessions where the robot movement follows pat-1, pat-2 and pat-3.

\section{F. Quantifying the Human Discomfort and Data Processing}

Previous studies have shown that the distance chosen by a human from a robot is a good measure of the discomfort when the human perceives near the robot [31] [32] [33] [34]. We observed that a similar distance measure could be utilized in our experiment to quantify discomfort. In the absence of the robot movement, participants performed a straight line reach in our task (ie. their UHT was straight). However, in the robotphase, when the robot moved close to them, the participant's reach deviated away from the robot. The inset plot in Fig. 2(a) shows a sample participant trajectory in the robot-phase when the robot movement patterns are pat-1 (blue traces), pat-2 (green traces) and pat-3 (red traces). We thus hypothesized that the 'trajectory deviation' exhibited by a participant, defined by the difference of the abscissa of the trial's ending point and the starting point, can serve as a behavioral measure of the discomfort perceived by the participant. We found a strong correlation between the trajectory deviation and reported anxiety by the participants in the SAM questionnaire (see results and Fig. 5), corroborating this hypothesis. 


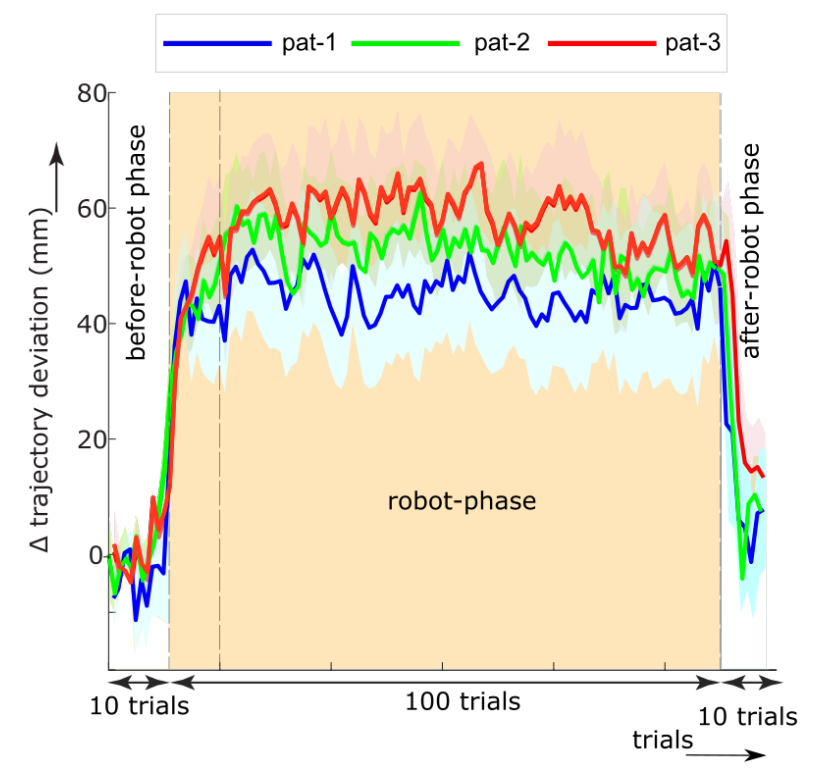

Figure 6. Trajectory deviation: The plot shows the across participant average (solid lines) and standard error (shaded regions) of the trajectory deviations across the three phases, when the robot performs pat-1 (blue data), pat-2 (green data) and pat-3(red data). Note that all the plotted data groups are not normal across participant, and have been accordingly treated with the appropriate statistics (described in the text). However, we choose to still show the data with a mean and standard error here for the purpose of visual clarity.

We also recorded electromyography (EMG) and photoplethysmography (PPG) from the participants in our study expecting to measure the physiological arousal felt by the participants due to their discomfort with the robot. EMG was recorded using non-invasive wireless sensors (Del-sys Trigno Wireless EMG System) from the participant's right and left biceps. PPG was recorded for 15 participants from the middle finger of the participant's left hand. PPG measures the blood flow and is known to show a decrease in amplitude, as well as increasing in pulse frequency when a person is aroused. However, we could not find any significant trend in either of these measurement across participants and through our experiment, and hence will not detail them in this manuscript

hereafter.

\section{RESULTS}

\section{A. Trajectories Deviation vs Discomfort}

We start by checking that our trajectory deviation measure represents discomfort. Across our four sessions, we found a significant correlation $(\mathrm{r}=0.24, \mathrm{p}=0.042)$ between the anxiety indicated by the participants in the SAM questionnaire in a session and the average deviation they exhibited in that session (Fig 5), suggesting that the trajectory deviation is a measure of participant discomfort.

\section{B. Trajectories Deviations}

Fig. 6 shows the trajectory deviation exhibited by 18 (of the 21) participants with the robot movement patterns after the subtraction of their individual median trajectory deviation

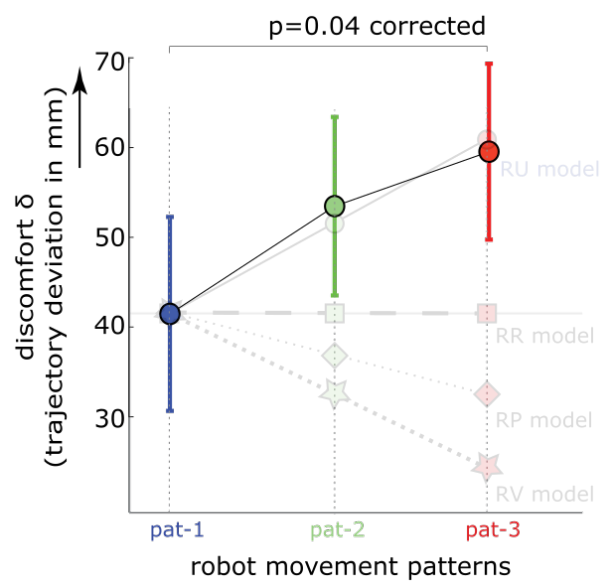

Figure 7. Model validation: Discomfort of the participants during our experi-ment, measured as the mean (solid line) and standard error (errorbars) in their trajectory deviation (in $\mathrm{mm}$ ) is plotted for the three robot patterns (different colours). The data plot has been superimposed on the model predictions from Fig. 4 to show that only the RU model can explain the human behavior in our experiment.

from the before robot phase. 3 participants were omitted from the data analysis due to a malfunction with the robot pattern (with 1 participant), and due to the inability to follow the sound cues ( 2 participants). Fig. 6 thus effectively plots the changes in trajectory deviation by the participants relative to their trajectory in their before robot phase.

We observe that all participants exhibit a significant increase in trajectory deviation when the robot starts moving in the robot-phase. In order to analyze the effect of the patterns and trials on their trajectory deviation, we performed a 2-way ANOVA (with first factor a s pattern, and s econd a s trials) on the trajectory deviations between the 11th and 100th trials in robot-phase. We omitted the first 10 t rials t o a llow f or the stabilization of the trajectories. We observed that the across participant distribution of the trajectory deviation did not follow a normal distribution (Shapiro-Wilk test of normality $\mathrm{p}<0.02$ ) for certain trials and hence we used the Aligned Rank Transform (ART) [35] as a preprocessing step before applying the ANOVA. The ANOVA revealed a significant main effect of patterns $(\mathrm{F}(2,4050)>169$, zp $<0.001)$, but not of trials $(\mathrm{F}(89,4050)=0.24, \mathrm{p}>0.9)$ and showed no significant interaction between the two factors $(\mathrm{F}(178,4050)=0.2$, $\mathrm{p}>0.9)$. The ANOVA result shows that after the initial 10 trials, participants maintain similar trajectory deviations for the rest of the robot-phase. This allowed us to quantify the trajectory deviations for the three robot patterns by the median trajectory deviation within the 11th and 100th trials of the robot-phase.

\section{Comparison with Model Predictions}

The across participant median trajectory deviation change is shown in Fig. 7. The deviation is superimposed over the model plot from Fig. 4. The trajectory deviation changes with each pattern were observed to be normal ( $p>0.56$, Shapiro-Wilk test) across the participants, with a value of $41.46 \pm 10.8 \mathrm{SE} \mathrm{mm}$ 
(SE standing for standard error) with pat-1, 53.4650 $\pm 9.9 \mathrm{SE}$ $\mathrm{mm}$ with pat-2 and 59.5400 $\pm 9.8 \mathrm{SE} \mathrm{mm}$ with pat-3. A 1way repeated measures ANOVA showed significant effect of robot pattern on the participants trajectory deviation change $(\mathrm{F}(2,17)=4.12, \mathrm{p}=0.020)$. A post-hoc T-test revealed a significant higher trajectory deviation for pat-3, compared to pat-1 $(\mathrm{p}=0.04$, Bonferonni corrected).

Comparing the trajectory deviation with our models in Fig. 7, we find that only our RU model can explain this data. The results thus show that the uncertainty in the robot movement was the fundamental determinant of human discomfort in our experiment.

\section{Questionnaire}

We asked participants to score three emotions in our sessions. As the scores were non-normal across participants in almost all the sessions, we considered a Kruskal Wallis test to look for a trend across the sessions. We observed no changes in feeling of 'control', 'anxiety' or 'happiness' across the movement patterns (chi-s q $<1.57, \mathrm{p}>0.46$ ).

However, a closer look at the values showed that, only the anxiety score showed a tendency of modulation across the sessions. The across participant anxiety score was less for pat1, compared to the average scores reported for pat-2 and pat3 ( $\mathrm{p}=0.06$, Wilcoxon Signed Rank test) by the participants. Furthermore, the anxiety scores correlated with the trajectory deviations exhibited by the participants (Fig. 5).

\section{DISCUSSION}

In this study, we examined the behaviors of humans working near a robot, to examine how different features of the robot movement influenced the discomfort felt by the human. Motivated by previous studies, we considered four models of discomfort. Our results show that the RU model, which considers human discomfort to increase with the movement uncertainty of the robot, best explains the human behaviors in our study.

This result is not completely unexpected given that previous studies in social neuroscience [21] as well as robot trust [11] [22] [23] have observed that the 'trust' felt by a human towards interacting agents is influenced by the predictability of the agent's behaviors, in regard to their decision making, task failures and errors. Here we show, probably for the first time using behavioral measures, that uncertainty is also critical at the level of robot movement trajectories, and influences the comfort felt with robots.

It is important to note that the human discomfort we observe here is modulated not by the robot movement uncertainty per se, but rather by the perceived robot movement uncertainty by the human. In our study, the two are similar but this may not always be the case. The perceived movement uncertainty is a consequence of both the robot movement uncertainty as well as the uncertainty in the perception of the robot movements by a human. For example, even a robot performing a regular movement pattern (with minimal movement uncertainty) may be considered uncomfortable inside a dark room, or when behind the human, or if the human is without her/his eye glasses, because in all these cases, the perception of the robot motion is limited, making them uncertain to the human. Corresponding to this, previous robot approach studies have popularly found that humans prefer that robot do not approach them from behind [15] [6].

Human perception is also constrained by the neural processing delays, thus making movements prediction more difficult (and hence more uncertain) when the time available for processing an observed robot is less. This is typically the case when a robot is moving fast. Movement uncertainty can therefore explain why you would be more uncomfortable with a fast moving robot, than a slow moving one even if they are making the same movements [14] [36]. In our experiment however, this was not the case because the faster (in terms of average speed) robot was more regular in movements than the slower one.

Finally, it is well known that humans can learn and develop models to explain the behaviors of partners they interact with [17] [21], a skill that is probably fundamental to our social interactions. It is thus expected that perceived uncertainty can change with time as the human starts to slowly understand the movements made by the robot. The perceived uncertainty related partner models can explain why a robot, that does not perform a task well, may still be regarded as trustworthy if the errors it makes are predictable [8], and how the physical appearance of robot can influence the trust felt towards it. For example, several studies have reported that humans are more accepting towards a humanoid robot than a non-humanoid one [8] [11]. This is probably because humans have a good understanding of the movement abilities and movement limitations of the human form (making the humanoid behaviors less uncertain), and hence more trustworthy. Furthermore, the ease of understanding the uncertainty in robot movements can explain observations where human participants have found to be more comfortable with a passive following robot (that is easier to understand) than an active robot that is helping them in the task [37] [38].

To conclude, we show using an empirical experiment that the perceived uncertainty in a robot's movement is a key determinant of how much humans are comfortable with them. While there are arguably other environmental, appearance and task related factors, we believe that many factors being currently considered to affect comfort and/or trust with robots can in fact be explained as being caused by the perceived uncertainty in the movements (or decisions, and errors) exhibited by a robot. We gave examples of some of these cases above. However, further in-depth analysis, of how movement uncertainties in a robot behavior are perceived and learnt by humans, is required to clarify this issue further.

\section{REFERENCES}

[1] B. V. et al., "Variable impedance actuators: A review," Robotics and Autonomous Systems, vol. 61, no. 12, pp. $1601-1614$, Dec 2013. [Online]. Available: http://www.sciencedirect.com/science/article/pii/S0921889013001188 
[2] P. Kazanzides, "Safety design for medical robots," in Proceedings of Annual International Conference of the IEEE Engineering in Medicine and Biology Society. IEEE, 2009, pp. 7208-7211.

[3] G. Ganesh, A. Albu-Schäffer, M. Haruno, M. Kawato, and E. Burdet, "Biomimetic motor behavior for simultaneous adaptation of force, impedance and trajectory in interaction tasks," in Proceedings of IEEE International Conference on Robotics and Automation. IEEE, 2010, pp. 2705-2711.

[4] S. Haddadin, A. Albu-Schäffer, A. D. Luca, and G. Hirzinger, "Collision detection and reaction: A contribution to safe physical human-robot interaction," in Proceedings of IEEE/RSJ International Conference on Intelligent Robots and Systems. IEEE, 2008, pp. 3356-3363.

[5] Y. Li, G. Ganesh, N. Jarrassé, S. Haddadin, A. Albu-Schäffer, and E. Burdet, "Force, impedance, and trajectory learning for contact tooling and haptic identification," IEEE Transactions on Robotics, vol. 34, no. 5, pp. 1170-44, Oct 2018. [Online]. Available: https://ieeexplore.ieee.org/abstract/document/8362715

[6] E. Sisbot, L. Marin-Urias, R. Alami, and T. Siméon, "A human aware mobile robot motion planner," Robotics, IEEE Transactions on, vol. 23, no. 5, pp. 874-883, Nov 2007.

[7] V. Rajamohan, C. Scully-Allison, S. Dascalu, and D. Feil-Seifer, "Factors influencing the human preferred interaction distance," in Proceedings of IEEE International Conference on Robot and Human Interactive Communication, 2019, pp. 1-7.

[8] T. Sanders, K. E. Oleson, D. R. Billings, J. Y. Chen, and P. A. Hancock, "A model of human-robot trust: Theoretical model development," in Proceedings of the Human Factors and Ergonomics Society Annual Meeting, vol. 55, no. 1, Sep 2011, pp. 1432-1436. [Online]. Available: https://doi.org/10.1177/1071181311551298

[9] D. Li, P.-L. P. Rau, and Y. Li, "A cross-cultural study: Effect of robot appearance and task," International Journal of Social Robotics, vol. 2, no. 2, pp. 175-186, Jun 2010.

[10] R. Parasuraman, T. Sheridan, and C. Wickens, "Situation awareness, mental workload, and trust in automation: Viable, empirically supported cognitive engineering constructs," Journal of Cognitive Engineering and Decision Making, vol. 2, pp. 140-160, Jul 2008.

[11] M. Lewis, K. Sycara, and P. Walker, "The role of trust in humanrobot interaction," in Foundations of Trusted Autonomy, ser. Studies in Systems, Decision and Control, A. H., S. J., and R. D., Eds. Springer, 2018, vol. 17, pp. 135-159.

[12] D. M. Rousseau, S. B. Sitkin, R. S. Burt, and C. Camerer, "Not so different after all: A cross-discipline view of trust," Academy of Management Review, vol. 23, no. 3, pp. 393-404, Jul 1998.

[13] B. Muir and N. Moray, "Trust in automation. part ii. experimental studies of trust and human intervention in a process control simulation," Ergonomics, vol. 39, pp. 429-60, Apr 1996.

[14] M. Koppenborg, P. Nickel, B. Naber, A. Lungfiel, and M. Huelke, "Effects of movement speed and predictability in human-robot collaboration," Human Factors and Ergonomics in Manufacturing \& Service Industries, vol. 27, no. 4, pp. 197-209, May 2017.

[15] K. Koay, K. Dautenhahn, S. Woods, and M. Walters, "Empirical results from using a comfort level device in human-robot interaction studies," in Proceedings of ACM/IEEE International Conference on Human-Robot Interaction, Jan 2006, pp. 194-201.

[16] R. Johansson, G. Westling, A. Bäckström, and J. Flanagan, "Eye-hand coordination in object manipulation," The Journal of neuroscience : the official journal of the Society for Neuroscience, vol. 21, no. 7, pp. 691732, Oct 2001

[17] A. Takagi, G. Ganesh, T. Yoshioka, M. Kawato, and E. Burdet, "Physically interacting individuals estimate the partner's goal to enhance their movements," Nature Human Behaviour, vol. 1, no. 3, pp. 23973374, Mar 2017. [Online]. Available: https://doi.org/10.1038/s41562017-0054

[18] G. Ganesh, A. Takagi, R. Osu., T. Yoshioka, M. Kawato, and E. Burdet, "Two is better than one: Physical interactions improve motor performance in humans," Scientific Reports, vol. 4, p. 3824, Jan 2014. [Online]. Available: https://www.nature.com/articles/srep03824supplementary-information

[19] A. D. Dragan, S. Bauman, J. Forlizzi, and S. S. Srinivasa, "Effects of robot motion on human-robot collaboration," in Proceedings of ACM/IEEE International Conference on Human-Robot Interaction. ACM, 2015, pp. 51-58.
[20] A. D. Dragan, K. C. Lee, and S. S. Srinivasa, "Legibility and predictability of robot motion," in Proceedings of ACM/IEEE International Conference on Human-Robot Interaction. ACM, 2013, pp. 301-308.

[21] R. Axelrod, The Complexity of Cooperation: Agent-Based Models of Competition and Collaboration. Princeton: Princeton University Press, 1997.

[22] S. Lewandowsky, M. Mundy, and G. Tan, "The dynamics of trust: Comparing humans to automation," Journal of experimental psychology. Applied, vol. 6, pp. 104-23, Jul 2010.

[23] V. A. Riley, "Human use of automation," Ph.D. dissertation, University of Minneapolis, 1994.

[24] D. M. Wolpert, J. Diedrichsen, and J. R. Flanagan, "Principles of sensorimotor learning," Nature Reviews Neuroscience, vol. 12, pp. 739751, Dec 2011.

[25] Y.-w. Tseng, J. Diedrichsen, J. W. Krakauer, R. Shadmehr, and A. J. Bastian, "Sensory prediction errors drive cerebellum-dependent adaptation of reaching," Journal of Neurophysiology, vol. 98, no. 1, pp. 54-62, 2007.

[26] D. W. Franklin and D. M. Wolpert, "Computational mechanisms of sensorimotor control," Neuron, vol. 72, no. 3, pp. 425-442, Nov 2011.

[27] D. J. Reinkensmeyer, J. L. Emken, and S. C. Cramer, "Robotics, motor learning, and neurologic recovery," Annual Review of Biomedical Engineering, vol. 6, no. 1, pp. 497-525, 2004.

[28] L. Marchal-Crespo and D. J. Reinkensmeyer, "Review of control strategies for robotic movement training after neurologic injury," Journal of NeuroEngineering and Rehabilitation, vol. 6, no. 20, 2009

[29] Y. Li, G. Ganesh, N. Jarrassé, S. Haddadin, A. Albu-Schäffer, and E. Burdet, "Force, impedance, and trajectory learning for contact tooling and haptic identification," IEEE Transactions on Robotics, vol. 34, no. 5, pp. 1170-1182, Oct 2018.

[30] M. M. Bradley and P. J. Lang, "Measuring emotion: The self-assessment manikin and the semantic differential," Journal of Behavior Therapy and Experimental Psychiatry, vol. 25, no. 1, pp. 49-59, 1994.

[31] M. M. E. Neggers, R. H. Cuijpers, and P. A. M. Ruijten, "Comfortable passing distances for robots," in Social Robotics, S. S. Ge, J.-J. Cabibihan, M. A. Salichs, E. Broadbent, H. He, A. R. Wagner, and Á. Castro-González, Eds., vol. 11357. Cham: Springer International Publishing, 2018, pp. 431-440.

[32] M. Walters, K. Koay, S. Woods, D. S. Syrdal, and K. Dautenhahn, "Robot to human approaches: Preliminary results on comfortable distances and preferences," in AAAI Spring Symposium-Technical Report, Jan 2007.

[33] M. L. Walters, K. Dautenhahn, K. L. Koay, C. Kaouri, R. Boekhorst, C. Nehaniv, I. Werry, and D. Lee, "Close encounters: spatial distances between people and a robot of mechanistic appearance," in 5th IEEERAS International Conference on Humanoid Robots, Dec 2005, pp. 450455 .

[34] T. Kanda and H. Ishiguro, Human-Robot Interaction in Social Robotics. CRC Press, Mar 2017.

[35] J. Wobbrock, L. Findlater, D. Gergle, and J. Higgins, "The aligned rank transform for nonparametric factorial analyses using only anova procedures," May 2011, pp. 143-146.

[36] A. Wojciechowska, J. Frey, S. Sass, R. Shafir, and J. R. Cauchard, "Collocated human-drone interaction: Methodology and approach strategy," in Proceedings of ACM/IEEE International Conference on Human-Robot Interaction, 2019, pp. 172-181.

[37] A. Bussy, "Approche cognitive pour la représentation de l'interaction proximale haptique entre un homme et un humanoïde," $\mathrm{Ph} . \mathrm{D}$. dissertation, LIRMM - Laboratoire d'Informatique de Robotique et de Microélectronique de Montpellier, 2013.

[38] Y. Li, G. Carboni, F. Gonzalez, D. Campolo, and E. Burde, "Measuring emotion: The self-assessment manikin and the semantic differential," Nature Machine Intelligence, vol. 1, no. 1, pp. 36-43, Jun 2019. 